\title{
Síndrome da ressecção anterior do reto: fatores preditivos.
}

\section{Predictive factors of low anterior resection syndrome following anterior resection of the rectum.}

Fernanda leticia Cavalcante Miacci'; Camila Roginski Guetter, AcCBC-PR²; Paula Heroso Moreira²@; Maria Cristina Sartor, TCBC-PR; Mariane Christina Savio'; Antonio Baldin Júniori; Norton Luiz Nóbrega ${ }^{1}$

\section{R E S U M O}

\begin{abstract}
Objetivo: identificar fatores preditivos da síndrome da ressecção anterior do reto (SRAR) que podem contribuir para o seu diagnóstico e tratamento precoces. Métodos: estudo de coorte retrospectivo de pacientes submetidos à ressecção anterior do reto entre 2007 e 2017 no Serviço de Coloproctologia do Hospital de Clínicas da Universidade Federal do Paraná. Foram realizadas análises de curva ROC (Receiver Operating Characteristic Curve Analysis) ou COR (Característica de Operação do Receptor) para identificar os fatores preditivos da SRAR. Resultados: foram incluídos 64 pacientes com dados completos. A idade dos homens foi de $60,1 \pm 11,4$ anos e $37,10 \%$ eram do sexo masculino. Vinte pacientes $(32,26 \%)$ apresentaram SRAR. Os sintomas mais relatados foram evacuação incompleta (60\%) e urgência (55\%). Na análise univariada, a distância da anastomose à margem anal $(p<0,001)$, terapia neoadjuvante $(p=0,0014)$ e confecção de ileostomia no momento da ressecção $(p=0,0023)$ foram preditivos da SRAR. Análise da curva ROC mostrou um ponto de corte de $6,5 \mathrm{~cm}$ na distância da anastomose à margem anal como preditor da SRAR. Conclusão: distância entre anastomose e margem anal, história de terapia neoajuvante e confecção de estoma são condições que podem ajudar a predizer o desenvolvimento da SRAR. A orientação e o envolvimento na educação do paciente, bem como, o manejo precoce podem reduzir potencialmente o impacto desses sintomas na qualidade de vida dos pacientes.
\end{abstract}

Descritores: Cirurgia Colorretal. Complicações Pós-Operatórias. Neoplasias Retais. Anastomose Cirúrgica. Estomas Cirúrgicos.

\section{INTRODUÇÃO}

$P$ ara o Brasil, estimam-se 17.380 casos novos de câncer de cólon e reto em homens e 18.980 em mulheres para cada ano do biênio 2018-2019. Esses valores correspondem a um risco estimado de 16,83 casos novos a cada 100 mil homens e 17,90 para cada 100 mil mulheres. É o terceiro câncer mais frequente em homens e o segundo entre as mulheres ${ }^{1}$.

Os principais objetivos do tratamento cirúrgico para pacientes com câncer do reto são a excisão completa do tumor com margem cirúrgica adequada, preservação esfincteriana e baixas taxas de morbidade e mortalidade? .

Apesar da evolução da técnica cirúrgica e atenção à preservação da inervação pélvica, muitos pacientes desenvolvem alterações urinárias, sexuais e intestinais graves após a ressecção anterior do reto (RAR). O conjunto destas alterações é denominado síndrome pós-ressecção anterior do reto (SRAR) ou LARS, do inglês "low anterior resection syndrome" ${ }^{3}$.
Os sintomas da SRAR incluem aumento da frequência evacuatória, fezes de consistência líquida, impactação fecal, urgência e incontinência fecal. Esses sintomas podem ocorrer em até $80 \%$ dos casos pós-RAR 4 . O início dos sintomas ocorre logo após o restabelecimento do trânsito intestinal, são mais intensos nos primeiros meses, e podem melhorar nos primeiros dois anos, quando atingem uma estabilidade e tornam-se uma condição crônica ${ }^{5}$.

$$
\text { O diagnóstico da SRAR é }
$$

predominantemente clínico e, para a avaliação dos resultados funcionais após RAR, comumente são utilizadas ferramentas que avaliam a incontinência fecal, dado o impacto deste sintoma na qualidade de vida do paciente, como por exemplo, o WexnerJorge score ${ }^{6}$. Duas ferramentas, o Instrumento de Função Intestinal do Memorial Sloan Kettering Cancer Center (MSKCC) (BFI) e o escore LARS $^{7}$, foram introduzidas na tentativa de identificar e quantificar o impacto dos sintomas na qualidade de vida pós-RAR. 
Com o objetivo de identificar a SRAR nos pacientes submetidos à RAR do reto no Serviço de Coloproctologia do Complexo Hospital de Clínicas da Universidade Federal do Paraná (CHC/UFPR), o presente trabalho busca determinar a incidência dos sintomas de SRAR e identificar fatores preditivos para desenvolvimento da síndrome.

\section{MÉTODOS}

O projeto foi aprovado pelo Comitê de Ética em Pesquisa do Hospital de Clínicas da Universidade Federal do Paraná (número do Certificado de Apresentação para Apreciação Ética - CAAE: 89684218.0.0000.0096).

Trata-se de um estudo de coorte longitudinal. Os dados do estudo foram obtidos de forma retrospectiva a partir dos prontuários de pacientes submetidos à RAR no Serviço de Coloproctologia do Hospital de Clínicas da Universidade Federal do Paraná (HC-UFPR), no período de janeiro de 2007 a dezembro de 2017.

Inicialmente foram selecionados 110 pacientes em que a operação cadastrada foi de retossigmoidectomia. Foram excluídos 48 pacientes, 33 por terem sido submetidos a outros procedimentos diferentes do cadastrado e 15 que preencheram os critérios de exclusão adotados, que foram: permanência do estoma de derivação de trânsito, prontuários incompletos, doença inflamatória intestinal, doença de Hirschsprung e recidiva pélvica precoce do tumor do reto (Figura 1).

A fim de avaliar a possível interferência de comorbidades na incidência de SRAR, utilizou-se o Charlson Comorbidity Index $(\mathrm{CCl})^{9}$. Foi considerado o relatório de descrição cirúrgica para identificar o tipo de cirurgia realizada e a distância da anastomose à margem anal. Para a avaliação do estadiamento $\mathrm{TNM}^{10}$, foi utilizado o exame anatomopatológico da peça cirúrgica.

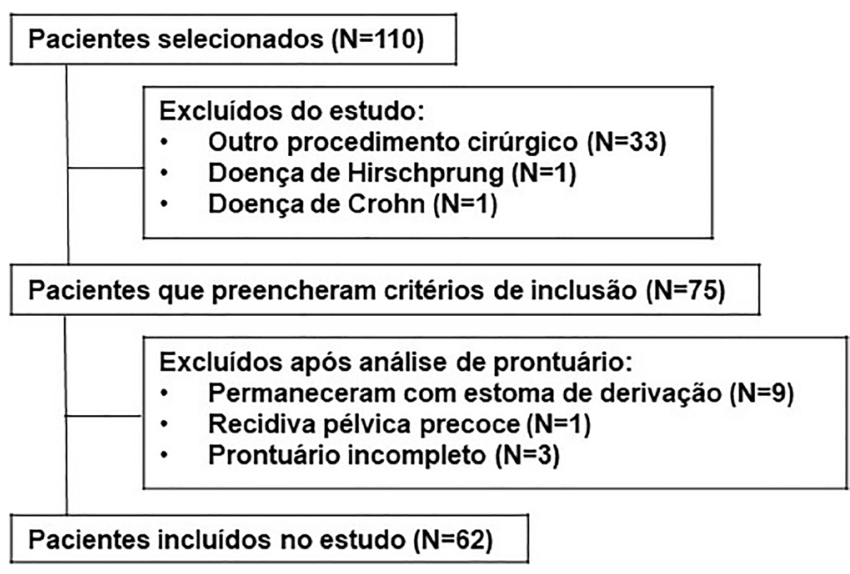

Figura 1. Método de inclusão.

Finalmente permaneceram no estudo 62 pacientes, que foram divididos em dois grupos: com SRAR e sem SRAR. A partir daí, foi aplicada estatística inferencial nos dois grupos.

Os dados coletados foram então analisados por meio do software estatístico $\mathrm{R}$ ( $\mathrm{R}$ Core Team (2018). R: A language and environment for statistical computing. R Foundation for Statistical Computing, Vienna, Austria11. Para a análise descritiva, as medidas de tendência central e de dispersão foram expressas em médias e desvio padrão (média $\pm D P$ ) para as variáveis contínuas de distribuição normal e em medianas e intervalo interquartil (mediana, 25\%-75\%) para as de distribuição não normal. As variáveis categóricas e ordinais foram expressas em frequências absolutas e relativas. O teste foi aplicado para determinação de normalidade das amostras.

$\mathrm{Na}$ análise univariada dos dados, foram utilizados os testes de $\mathrm{t}$ de Student para variáveis dependentes contínuas normais e Mann-Whitney para variáveis dependentes contínuas não normais. Os testes de chi-quadrado e Fisher foram utilizados para variáveis dependentes binárias ou categóricas. Finalmente, análise através de curva ROC (Receiver Operating Characteristic Curve Analysis) foi realizada para identificar a distância da anastomose à margem anal preditora para sintomas de SRAR. Foi considerado nível de significância de 5\% para este estudo. 


\section{RESULTADOS}

Dos 62 pacientes incluídos, 39 (63\%) eram do sexo feminino. A média de idade foi 60,1 anos, variando de 33 a 85 anos. A distância média da anastomose em relação à borda anal foi de $9,57 \mathrm{~cm}$, variando de $2,0 \mathrm{a}$ $15 \mathrm{~cm}$ (Tabela 1).

Tabela 1. Características dos pacientes incluídos no estudo.

\begin{tabular}{|c|c|}
\hline Var & \\
\hline Idade (anos), média \pm desvio padrão & $60,1 \pm 11,4$ \\
\hline \multicolumn{2}{|l|}{ Sexo* } \\
\hline Masculino & $23(37,10 \%)$ \\
\hline Feminino & $39(62,90 \%)$ \\
\hline \multicolumn{2}{|l|}{ Tabagismo* } \\
\hline Ex & $14(22,58 \%)$ \\
\hline Atual & $9(14,52 \%)$ \\
\hline Nunca & $39(62,90 \%)$ \\
\hline \multicolumn{2}{|l|}{ Etilismo* } \\
\hline Ex & $5(8,06 \%)$ \\
\hline Atual & $1(1,61 \%)$ \\
\hline Nunca & $56(90,32 \%)$ \\
\hline \multicolumn{2}{|l|}{ Pontos $\mathrm{CCl}^{* *}$} \\
\hline 0 & $2(3,22 \%)$ \\
\hline 1 & $5(8,06 \%)$ \\
\hline 2 & $16(25,81 \%)$ \\
\hline 3 & $15(24,19 \%)$ \\
\hline 4 & $5(8,06 \%)$ \\
\hline 5 & $11(17,74 \%)$ \\
\hline 6 & $6(9,68 \%)$ \\
\hline 7 & $1(1,61 \%)$ \\
\hline 8 & $1(1,61 \%)$ \\
\hline \multicolumn{2}{|l|}{ Cirurgia pélvica prévia* } \\
\hline Sim & $13(20,97 \%)$ \\
\hline Não & $49(79,03 \%)$ \\
\hline \multicolumn{2}{|l|}{ Diagnóstico para indicação cirúrgica* } \\
\hline Neoplasia & $42(66,13 \%)$ \\
\hline Patologia não maligna & $20(32,26 \%)$ \\
\hline \multicolumn{2}{|l|}{ Local do tumor, se neoplasia* } \\
\hline Reto distal & $1(2,44 \%)$ \\
\hline Reto médio & $14(34,15 \%)$ \\
\hline Reto proximal & $9(21,95 \%)$ \\
\hline Junção retossigmoide & $4(9,76 \%)$ \\
\hline Cólon sigmoide & $13(31,71 \%)$ \\
\hline \multicolumn{2}{|l|}{ Estadiamento TNM, se neoplasia* } \\
\hline TO & $3(7,32 \%)$ \\
\hline T1 & $4(9,76 \%)$ \\
\hline $\mathrm{T} 2$ & $14(34,15 \%)$ \\
\hline T3 & $15(36,58 \%)$ \\
\hline T4 & $5(12,19 \%)$ \\
\hline
\end{tabular}


...continuação

Variável

Neoadjuvância, se neoplasia*

Sim

$13(31,71 \%)$

Não

$28(68,29 \%)$

Tipo de anastomose*

Manual

$20(32,26 \%)$

Grampeada

$42(67,74 \%)$

Distância anastomose à margem anal $(\mathrm{cm})^{\dagger}$

$10,00(6,25-12,00)$

Confecção de estoma protetor*

$\operatorname{Sim}$

$31(50 \%)$

Não

$31(50 \%)$

Tempo pós-retossigmoidectomia para fechamento do estoma (meses) ${ }^{\dagger}$

7,36 (4,70-10,92)

* Frequência absoluta (frequência relativa); * * CCl: Charlson comorbidity index; ${ }^{+}$Mediana (intervalo interquartil 25\%-75\%).

As doenças que levaram à ressecção anterior do reto foram: doença diverticular do cólon sigmoide, fístula retovaginal pós-radioterapia, perfuração do sigmoide durante colonoscopia, prolapso do reto, megacólon chagásico, volvo do sigmoide, fístula sigmoidevesical e neoplasias do sigmoide distal, da junção retossigmoideana, do reto proximal, médio e distal.

Do total de 62 pacientes avaliados, 20 (32\%) tinham sintomas da SRAR. Todos os pacientes apresentavam mais de um sintoma, sendo as queixas mais comuns relacionadas ao trato gastrointestinal, à evacuação incompleta (60\%), à urgência evacuatória (55\%) e à alteração de hábito intestinal, como diarreia $(45 \%)$ e constipação (45\%). Sintomas não relacionados ao trato gastrointestinal foram menos frequentes, com quatro pacientes (20\%) com sintomas urinários e apenas um paciente (5\%) com queixas relacionadas à função sexual (Tabela 2).

A causa mais comum que levou à ressecção anterior do reto foi neoplasia maligna, em 42 pacientes (67\%), seguida pela doença diverticular, em dez pacientes (16\%) e megacólon chagásico em cinco (8\%).
Dos pacientes com neoplasia, as localizações mais comuns foram no reto médio, sigmoide distal e reto proximal, com $34 \%$, $31 \%$ e $22 \%$, respectivamente. Da estatística inferencial, os fatores que apresentaram relevância estatística para desenvolvimento da síndrome foram: realização de neoadjuvância ( $p=0,0014)$, a distância da anastomose da borda anal $(p<0,001)$ e a utilização de estoma de derivação $(p=0,0023)$ (Tabela 3).

A análise da curva ROC mostrou um ponto de corte de $6,5 \mathrm{~cm}$ na distância da anastomose em relação à borda anal, onde, abaixo desta altura, o risco de desenvolver a síndrome é maior (Figura 2).

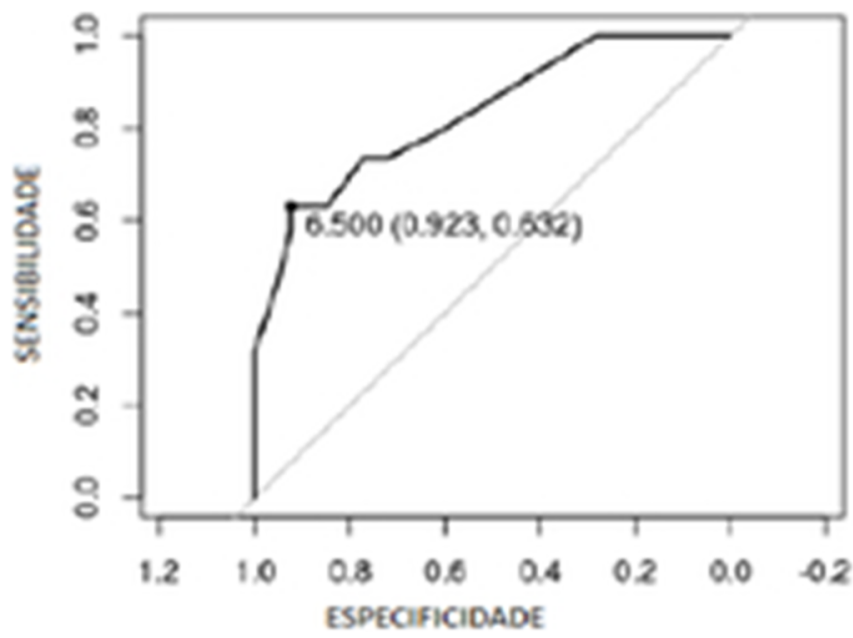

Figura 2. Curva ROC: área abaixo da curva: $0.831 ; 95 \%$ intervalo de confiança: 0.7191-0.9435. 
Tabela 2. Diagnóstico e tratamento de SRAR.

\begin{tabular}{|c|c|}
\hline Variável & \\
\hline \multicolumn{2}{|l|}{ Sintomas SRAR* } \\
\hline Sim & $20(32,26 \%)$ \\
\hline Não & $42(67,74 \%)$ \\
\hline \multicolumn{2}{|l|}{ Tipo de sintoma relatado** } \\
\hline Incontinência fecal & $7(35 \%)$ \\
\hline Urgência fecal & $11(55 \%)$ \\
\hline Evacuação incompleta & $12(60 \%)$ \\
\hline Diarreia & $9(45 \%)$ \\
\hline Constipação/Evacuação obstruída & $9(45 \%)$ \\
\hline Queixas sexuais & $1(5 \%)$ \\
\hline Sintomas urinários & $4(20 \%)$ \\
\hline Tempo pós-operatório até início dos sintomas (meses) ${ }^{\dagger}$ & $3.35(2,22-5,75)$ \\
\hline \multicolumn{2}{|l|}{ Diagnóstico de SRAR** } \\
\hline Sim & $15(24,19 \%)$ \\
\hline Não & $47(75,81 \%)$ \\
\hline \multicolumn{2}{|l|}{ Forma de diagnóstico** } \\
\hline Clínico & $13(86,67 \%)$ \\
\hline Clínico associado a exames complementares & $2(13,33 \%)$ \\
\hline \multicolumn{2}{|l|}{ Tipo de tratamento** } \\
\hline Fisioterapia pélvica apenas & $1(6,67 \%)$ \\
\hline Tratamento farmacológico apenas & $12(80 \%)$ \\
\hline Fisioterapia e tratamento farmacológico & $2(13,33 \%)$ \\
\hline \multicolumn{2}{|l|}{ Relato de melhora dos sintomas** } \\
\hline Sim & $11(73,33 \%)$ \\
\hline Não & $3(20 \%)$ \\
\hline Não relatado & $1(6,67 \%)$ \\
\hline \multicolumn{2}{|l|}{ Relato de melhora completa dos sintomas** } \\
\hline Sim & $6(40 \%)$ \\
\hline Não & $8(53,33 \%)$ \\
\hline Não relatado & $1(6,67 \%)$ \\
\hline
\end{tabular}

* SRAR: síndrome de ressecção anterior do reto; * * frequência absoluta (frequência relativa); ${ }^{+}$mediana (intervalo interquartil $25 \%-75 \%)$.

As variáveis sexo, idade, tabagismo, história de etilismo, história prévia de cirurgia pélvica, comorbidades $(\mathrm{CCl})$, estadiamento, tipo da anastomose e localização do tumor não foram preditivos para o desenvolvimento de SRAR.

\section{DISCUSSÃO}

A etiologia da síndrome da ressecção anterior do reto é multifatorial. Acredita-se que a SRAR esteja relacionada à diminuição do reservatório retal, à denervação do cólon esquerdo e do reto durante sua mobilização ${ }^{12}$ e à potencial lesão esficteriana durante a construção da anastomose grampeada ${ }^{13}$.
A incidência dos sintomas após ressecção anterior do reto de $32 \%$ nesta casuística é compatível com a literatura ${ }^{14}$. A dificuldade em estabelecer a real incidência da síndrome está no vasto espectro de sintomas e na falta de consistência na busca ativa de fatores de risco ${ }^{15}$.

Da mesma maneira que o relatado em outros trabalhos ${ }^{16-18}$, neste não foi encontrada significância estatística entre a incidência de SRAR e o sexo, embora Liu et al. ${ }^{19}$ tenham relatado o sexo feminino como fator de risco independente para a síndrome e Gadan et al. ${ }^{20}$ tenham descrito uma maior frequência de incontinência e flatos no sexo feminino. 
Tabela 3. Análise univariada comparando pacientes com e sem sintomas de SRAR.

\begin{tabular}{|c|c|c|c|}
\hline Variável & Com SRAR*(N=20) & Sem SRAR* $(N=42)$ & $p$ \\
\hline Sexo masculino** & $5(25 \%)$ & $18(42,86 \%)$ & 0,2804 \\
\hline 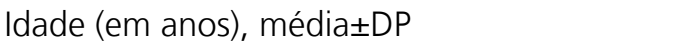 & $61,86 \pm 11,32$ & $59,31 \pm 11,51$ & 0,4143 \\
\hline Tabagismo** & & & 1,00 \\
\hline Ex & $4(20 \%)$ & $10(23,81 \%)$ & \\
\hline Atual & $3(15 \%)$ & $6(14,29 \%)$ & \\
\hline Nunca & $13(65 \%)$ & $26(61,9 \%)$ & \\
\hline Etilismo** & & & 1,00 \\
\hline Ex & $2(10 \%)$ & $3(7,14 \%)$ & \\
\hline Atual & $0(0 \%)$ & $1(2,38 \%)$ & \\
\hline Nunca & $18(90 \%)$ & $38(90,48 \%)$ & \\
\hline História de cirurgia pélvica prévia** & $7(35 \%)$ & $6(14,29 \%)$ & 0,1237 \\
\hline $\mathrm{CCl}^{* * *}$ & $3(2,5-5)$ & $3(2-5)$ & 0,2789 \\
\hline Estadiamento TNM, se neoplasia** & & & 0,0773 \\
\hline T0 & $3(18,75 \%)$ & $0(0 \%)$ & \\
\hline T1 & $1(6,25 \%)$ & $3(12 \%)$ & \\
\hline $\mathrm{T} 2$ & $6(37,5 \%)$ & $8(32 \%)$ & \\
\hline T3 & $6(37,5 \%)$ & $9(36 \%)$ & \\
\hline $\mathrm{T} 4$ & $0(0 \%)$ & $5(20 \%)$ & \\
\hline Realização de neoajduvância, se neoplasia** & $10(62,5 \%)$ & $3(12 \%)$ & 0,0014 \\
\hline Tipo de anastomose ${ }^{* *}$ & & & 1,00 \\
\hline Manual & $6(30 \%)$ & $14(33,34 \%)$ & \\
\hline Grampeada & $14(70 \%)$ & $28(66,66 \%)$ & \\
\hline Distância anastomose - margem anal $(\mathrm{cm})^{\dagger}$ & $5(3-10)$ & $12(9-15)$ & $<0,001^{\S}$ \\
\hline Distância anastomose - margem anal $(\mathrm{cm})^{* *}$ & & & $<0,001^{\S}$ \\
\hline$<8 \mathrm{~cm}$ & $14(73,68 \%)$ & $9(23,08 \%)$ & \\
\hline$>8 \mathrm{~cm}$ & $5(26,32 \%)$ & $30(76,92 \%)$ & \\
\hline Confecção de estoma protetor** & $16(80 \%)$ & $15(35,71 \%)$ & $0,0023^{\S}$ \\
\hline Tempo até fechamento do estoma (meses) ${ }^{\dagger}$ & $7,61(5,93-8,86)$ & $7,36(4-11,83)$ & 0,9213 \\
\hline Local do tumor, se neoplasia** & & & $0,0056^{\S}$ \\
\hline Reto distal & $0(0 \%)$ & $1(4 \%)$ & \\
\hline Reto médio & $10(62,5 \%)$ & $4(16 \%)$ & \\
\hline Reto proximal & $4(25 \%)$ & $5(20 \%)$ & \\
\hline Junção retossigmoide & $1(6,25 \%)$ & $3(12 \%)$ & \\
\hline Cólon sigmoide & $1(6,25 \%)$ & $12(48 \%)$ & \\
\hline
\end{tabular}

* SRAR: síndrome de ressecção anterior do reto; ** frequência absoluta (frequência relativa); *** CCl: Charlson comorbidity index; ${ }^{+}$mediana (intervalo interquartil 25\%-75\%); ` análises nas quais foi observada significância estatística $(p<0,05)$.

Quanto à idade, encontrou-se uma média global de 60,1 anos, compatível com os dados de Scheer et al. ${ }^{15}$ e Ekkarat et al. ${ }^{16}$. Devido a esta média de idade se associar com comorbidades pelo envelhecimento, foi feita a avaliação de risco de desenvolver SRAR com o índice $\mathrm{CCI}^{9}$. Não houve significância estatística entre eles. Não foram encontrados outros relatos na literatura.
Quando investigadas outras condições e hábitos de vida, como tabagismo e etilismo, estes não se mostraram estatisticamente relevantes. Não foram encontrados outros relatos na literatura para comparação.

Devido ao fato de cirurgias pélvicas poderem comprometer negativamente a inervação da região e atuar como fator confundidor no desenvolvimento 
da SRAR, foi comparada a incidência de SRAR em pacientes com e sem cirurgias pélvicas prévias. Não houve relevância estatística, o que foi compatível com a literatura ${ }^{16}$.

Nesse estudo, a casuística constitui-se de pacientes com doenças benignas e malignas, e os relatos de literatura normalmente utilizam pacientes submetidos à RAR por neoplasia, limitando, assim, a comparação deste com outros trabalhos. A maior parte dos pacientes (67\%) era portadora de neoplasia colorretal. Destes, 40\% desenvolveram a síndrome, o que é compatível com a literatura ${ }^{14}$.

A proporção de pacientes que sofrem dessa síndrome parece aumentar à medida que o nível da anastomose se aproxima do esfíncter anal. A ressecção anterior do reto com anastomose coloanal pode estar associada à maior incidência de incontinência. Após a cirurgia, a pressão anal de repouso diminui significativamente e não se recupera no curso do primeiro ano. O segundo efeito importante sobre a função do esfíncter anal é o possível desaparecimento do reflexo inibitório retoanal (RIRA), dependendo do nível de ressecção retal ${ }^{14}$. Embora o tratamento dos tumores do reto médio e distal necessite de cirurgia com anastomoses mais próximas da borda anal, a simples localização do tumor mostrou, neste trabalho, apenas uma tendência à significância estatística $(p=0,056)$. Curiosamente, quando analisada a altura da anastomose colorretal, os dados revelam como fator de risco para o desenvolvimento de SRAR, anastomoses realizadas até $6,5 \mathrm{~cm}$ da borda anal $(p<0.001)$. Em outros trabalhos, o valor da distância em relação à margem anal foi de $5 \mathrm{~cm}^{16,20}$.

Apesar de nesta casuística não ter sido encontrada significância estatística quando comparada anastomose mecânica e manual com a incidência da síndrome $(p=1,00)$, há divergências na literatura sobre o assunto. Na revisão de Pucciani ${ }^{14}$, o autor relatou maior incidência da síndrome com a anastomose grampeada, pois há danos diretos ao esfíncter anal resultantes da dilatação anal devido à introdução transanal do grampeador, como mostrado por exames de ultrassonografia endoanal ${ }^{13}$. Até $18 \%$ dos pacientes que se submetem à ressecção anterior com anastomose baixa grampeada apresentam evidências de lesão do esfíncter interno no longo prazo ${ }^{21}$.

Nesta casuísta, e compatível com a literatura $3-5,7,8,14,15,22-24$, a neoadjuvância mostrou-se como importante fator de risco para o desenvolvimento da síndrome $(p=0.014)$, assim como, os piores resultados relacionados à frequência e intensidade dos sintomas da SRAR quando comparados a pacientes tratados apenas por cirurgia. O mecanismo parece estar relacionado à lesão direta dos nervos e à fibrose pélvica induzida pela irradiação pélvica. Este tratamento também pode causar disfunções sexuais e urinárias devido à lesão nervosa ${ }^{25}$.

Por último, mas não menos importante, a ultrassonografia endoanal mostra mais cicatrizes dos esfíncteres anais em pacientes que tiveram radioterapia para terapia neoadjuvante ou adjuvante do que em pacientes não irradiados ${ }^{26}$.

Tem sido demonstrado que a ileostomia para desvio do trânsito pode causar alterações estruturais e funcionais no segmento do cólon abaixado e do coto retal. Estes incluem atrofia da parede intestinal e da musculatura do esfíncter anal, atrofia das vilosidades, inflamação da mucosa levando à colite de desuso. Postulase que essas alterações podem contribuir para os sintomas de SRAR após a restauração do trânsito intestinal. Há divergência na literatura sobre este assunto. No trabalho de Floodeen et al. ${ }^{27}$ não foi observada alteração significativa da função anorretal do primeiro ao quinto ano de seguimento em relação a pacientes que não haviam feito o estoma para derivação do trânsito. 
No entanto, os mesmos pacientes que foram aleatoriamente designados para um estoma temporário mais frequentemente relataram incontinência para flatos e fezes líquidas, bem como, uma maior pontuação total no escore LARS quando acompanhados por um período de 12 anos $^{20}$. A análise desta casuística mostrou que a realização do estoma de derivação constitui fator de risco para desenvolvimento da síndrome $(p=0.0023)$, e o início dos sintomas ocorreu, em média, 5,69 meses após o re-estabelecimento do trânsito intestinal.

Na revisão sistemática com 128 artigos por Keane et al. ${ }^{3}$, a incontinência fecal foi o sintoma mais frequente, sendo relatado em $97 \%$ dos estudos utilizados nessa metanálise. O mesmo achado foi referido na revisão de Bryant et al. ${ }^{28}$, que mostrou $71 \%$ dos pacientes com esta queixa. Na casuísta do Hospital de Clínicas do Paraná, dos sintomas gastrointestinais, a evacuação incompleta foi o sintoma mais frequente $(60 \%)$, seguido por urgência evacuatória (55\%), diarreia (45\%), constipação (45\%) e incontinência fecal (35\%). A dificuldade para avaliação da frequência dos sintomas ocorre pela falta de uniformização de ferramentas para diagnóstico da SRAR.

Os sintomas não relacionados ao trato gastrointestinal foram menos frequentes nesta casuística, com quatro (20\%) pacientes com sintomas urinários e apenas um (5\%) paciente com queixas relacionadas à função sexual. Apesar da forte associação entre os sintomas sexuais e urinários e a SRAR, a literatura sobre o assunto é escassa.

A principal limitação do nosso estudo é ser retrospectivo, o que impossibilita o questionamento direto ao paciente em relação ao sintoma. Outras limitações são o número de pacientes e cirurgias realizadas por cirurgiões distintos do Serviço de Coloproctologia.

Concluímos, assim, que a incidência da SRAR no Serviço de Coloproctologia do Hospital de Clínicas da Universidade Federal do Paraná, no período de janeiro de 2007 a dezembro de 2017, foi de 32\%, e os fatores de risco para desenvolver a síndrome foram: neoadjuvância, anastomoses abaixo de $6,5 \mathrm{~cm}$ da borda anal e confecção de estoma protetor.

A alta incidência dos sintomas da SRAR, mesmo em centros especializados, mostra que as alterações funcionais e estruturais causadas pela remoção do reto talvez possam ser minimizadas, mas não evitadas. Visto isso, torna-se importante a orientação, o envolvimento na educação do paciente e o diagnóstico precoce para estabelecer estratégia terapêutica que minimize os sintomas após a ressecção anterior do reto e o impacto desses sintomas na qualidade de vida dos pacientes.

\title{
A B S T R A C T
}

\begin{abstract}
Objective: to identify predictors of low anterior resection syndrome (LARS) that can contribute to its early diagnosis and treatment. Methods: we conducted a retrospective cohort study of patients undergoing anterior resection of the rectum between 2007 and 2017 in the Coloproctology Service of the Federal University of Parana Clinics Hospital. We performed Receiver Operating Characteristic Curve (ROC) analysis to identify LARS predictive factors. Results: we included 64 patients with complete data. The men's age was $60.1 \pm 11.4$ years and $37.10 \%$ were male. Twenty patients (32.26\%) had LARS. The most reported symptoms were incomplete evacuation (60\%) and urgency (55\%). In the univariate analysis, the distance from the anastomosis to the anal margin $(p<0.001)$, neoadjuvant therapy $(p=0.0014)$ and ileostomy at the time of resection $(p=0.0023)$ were predictive of LARS. The ROC curve analysis showed a $6.5 \mathrm{~cm}$ cut-off distance from the anastomosis to the anal margin as a predictor of LARS. Conclusion: distance between the anastomosis and the anal margin, neoadjuvant therapy history and preparation of stoma are conditions that can help predict the development of LARS. Guidance and involvement in patient education, as well as early management, can potentially reduce the impact of these symptoms on patients' quality of life.
\end{abstract}

Keywords: Colorectal Surgery. Postoperative Complications. Rectal Neoplasms. Anastomosis, Surgical. Surgical Stomas. 


\section{REFERÊNCIAS}

1. Instituto Nacional de Câncer José de Alencar Gomes da Silva. Coordenação de Prevenção e Vigilância. Estimativa 2018: incidência de câncer no Brasil. Rio de Janeiro: INCA; 2017.

2. Lopez-Kostner F, Lavery IC, Hool GR, Rybicki LA, Fazio VW. Total mesorectal excision is not necessary for cancers of the upper rectum. Surgery. 1998;124(4):612-7; discussion 617-8.

3. Keane C, Wells C, O'Grady G, Bissett IP. Defining low anterior resection syndrome: a systematic review of the literature. Colorectal Dis. 2017;19(8):713-22.

4. Martellucci J. Low anterior resection syndrome: a treatment algorithm. Dis Colon Rectum. 2016;59(1):79-82.

5. Ziv Y, Zbar A, Bar-Shavit Y, Igov I. Low anterior resection syndrome (LARS): cause and effect and reconstructive considerations. Tech Coloproctol. 2013;17(2):151-62.

6. Jorge JMN, Wexner SD. Etiology and management of fecal incontinence. Dis Colon Rectum. 1993;36(1):77-97.

7. Temple LK, Bacik J, Savatta SG, Gottesman L, Paty PB, Weiser MR, et al. The development of a validated instrument to evaluate bowel function after sphincter-preserving surgery for rectal cancer. Dis Colon Rectum. 2005;48(7):1353-65.

8. Emmertsen KJ, Laurberg S. Low anterior resection syndrome score: development and validation of a symptom-based scoring system for bowel dysfunction after low anterior resection for rectal cancer. Ann Surg. 2012;255(5):922-8.

9. Hall WH, Ramachandran R, Narayan S, Jani $A B$, Vijayakumar $\mathrm{S}$. An electronic application for rapidly calculating Charlson comorbidity score. BMC Cancer. 2004;4:94.

10. Weiser MR. AJCC 8th Edition: Colorectal Cancer. Ann Surg Oncol. 2018;25(6):1454-5.

11. The R Project for Statistical Computing [software]. 2019 Jul 05 [cited 2019]. Available from: https:// www.r-project.org/
12. Dulskas A, Smolskas E, Kildusiene I, Samalavicius NE. Treatment possibilities for low anterior resection syndrome: a review of the literature. Int J Colorectal Dis. 2018;33(3):251-60.

13. Ho YH, Tsang C, Tang CL, Nyam D, Eu KW, SeowChoen F. Anal sphincter injuries from stapling instruments introduced transanally: randomized, controlled study with endoanal ultrasound and anorectal manometry. Dis Colon Rectum. 2000;43(2):169-73.

14. Pucciani F. A review on functional results of sphinctersaving surgery for rectal cancer: the anterior resection syndrome. Updates Surg. 2013;65(4):257-63.

15. Scheer AS, Boushey RP, Liang $S$, Doucette $S$, O'Connor AM, Moher D. The long-term gastrointestinal functional outcomes following curative anterior resection in adults with rectal cancer: A systematic review and meta-analysis. Dis Colon Rectum. 2011;54(12):1589-97.

16. Ekkarat $\mathrm{P}$, Boonpipattanapong $\mathrm{T}$, Tantiphlachiva $\mathrm{K}$, Sangkhathat $S$. Factors determining low anterior resection syndrome after rectal cancer resection: a study in Thai patients. Asian J Surg. 2016;39(4):225-31.

17. Lange $M M$, den Dulk $M$, Bossema ER, Maas $C P$, Peeters KC, Rutten HJ, Klein Kranenbarg E, Marijnen CA, van de Velde CJ; Cooperative Clinical Investigators of the Dutch Total Mesorectal Excision Trial. Risk factors for faecal incontinence after rectal cancer treatment. Br J Surg. 2007;94(10):1278-84.

18. Nilsson S, Norlén BJ, Widmark A. A systematic overview of radiation therapy effects in prostate cancer. Acta Oncol. 2004;43(4):316-81.

19. Liu F, Guo P, Shen Z, Gao Z, Wang S, Ye Y. [Risk factor analysis of low anterior resection syndrome after anal sphincter preserving surgery for rectal carcinoma]. Chinese J Gastrointest Surg. 2017;20(3):289-94. Chinese.

20. Gadan S, Floodeen H, Lindgren R, Matthiessen P. Does a defunctioning stoma impair anorectal function after low anterior resection of the rectum for cancer? A 12-year follow-up of a randomized multicenter trial. Dis Colon Rectum. 2017;60(8):800-6. 
21. Farouk R, Duthie GS, Lee PW, Monson JR. Endosonographic evidence of injury to the internal anal sphincter after low anterior resection: longterm follow-up. Dis Colon Rectum. 1998;41(7):88891.

22. Campelo P, Barbosa E. Coloproctology Functional outcome and quality of life following. J Coloproctol. (Rio J.) [Online]. 2016 Dec [cited 2019];36(4):251-61. Available from: http:// www.scielo.br/scielo.php?script=sci_arttext\& pid=S223793632016000400251\&lng=en.

23. Emmertsen KJ, Chen TYT, Laurberg S. Functional results after treatment for rectal cancer. J Coloproctol. (Rio J.) [Online]. 2014 Mar [cited 2019];34(1):55-61. Available from: http://www. scielo.br/scielo.php?script= sci_arttext\&pid=\$223793632014000100055\&lng=en.

24. Martin ST, Heneghan HM, Winter DC. Systematic review of outcomes after intersphincteric resection for low rectal cancer. Br J Surg. 2012;99(5):603-12.

25. Buzatti KCLR, Petroianu A. Pathophysiological aspects of the low anterior resection syndrome for treatment of rectal cancer. Rev Col Bras Cir. 2017;44(4):397-402.
26. Pollack J, Holm T, Cedermark B, Holmström B, Mellgren A. Long-term effect of preoperative radiation therapy on anorectal function. Dis Colon Rectum. 2006;49(3):345-52.

27. Floodeen $H$, Lindgren $R$, Hallböök $O$, Matthiessen $P$. Evaluation of long-term anorectal function after low anterior resection: a 5-year follow-up of a randomized multicenter trial. Dis Colon Rectum. 2014;57(10):1162-8.

28. Bryant CLC, Lunniss PJ, Knowles CH, Thaha MA, Chan $\mathrm{CLH}$. Anterior resection syndrome. Lancet Oncol. 2012;13(9):e403-8.

Recebido em: 22/09/2019

Aceito para publicação em: 22/10/2019

Conflito de interesse: nenhum.

Fonte de financiamento: nenhuma.

\section{Endereço para correspondência:}

Paula Heroso Moreira

E-mail: paulaheroso@gmail.com fernanda.miacci@gmail.com

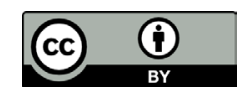

